\title{
Are we really seeking for equivalence? - The virtue of the robot is in technology
}

\author{
Aldo Brassetti ${ }^{1}$, Riccardo Mastroianni ${ }^{2}$, Giuseppe Simone ${ }^{1}$ \\ ${ }^{1}$ Department of Urology, "Regina Elena” National Cancer Institute of Rome, Rome, Italy; ${ }^{2}$ Department of Urology, "Sapienza” University of Rome, \\ Rome, Italy \\ Correspondence to: Riccardo Mastroianni. Department of Urology, “Sapienza” University of Rome, Rome, Italy. Email: ric.mastroianni@libero.it. \\ Provenance: This is an invited article commissioned by Section Editor Xiao Li (Department of Urology, Jiangsu Cancer Hospital \& Jiangsu Institute \\ of Cancer Research \& Nanjing Medical University Affiliated Cancer Hospital, Nanjing, China). \\ Comment on: Hussein AA, Elsayed AS, Aldhaam NA, et al. Ten-Year Oncologic Outcomes Following Robot-Assisted Radical Cystectomy: Results \\ from the International Robotic Cystectomy Consortium. J Urol 2019;202:927-35.
}

Submitted Aug 08, 2019. Accepted for publication Aug 15, 2019.

doi: $10.21037 /$ tau.2019.08.19

View this article at: http://dx.doi.org/10.21037/tau.2019.08.19

Radical cystectomy (RC) with pelvic lymph node dissection (PLND) and urinary diversion (UD) is the gold standard treatment for organ-confined muscle-invasive bladder cancer (MIBC) and recurrent non-muscle invasive disease (1). The open approach is still considered the reference option though the robot-assisted one has recently begone prevailing in western countries $(2,3)$, mainly because of the expected advantages of this minimally-invasive technology in terms of reduced morbidity. Nevertheless, the superiority of robotic surgery remains debated: while most of the authors agree with the finding that this approach reduces intraoperative blood loss and transfusions (4), all the available randomized controlled trials (RCTs) failed to prove any significant difference in minor and major complications rate, both at 30 and 90 days after surgery (5-9).

Cystectomy is indeed a complex procedure and most of its morbidity is ascribed to the reconstructive surgical step (10). Because of this, despite Pasadena Consensus Conference recommendations (11) and potentially undermining the expected benefits of laparoscopy, a nonnegligible share of surgeons still prefer an extracorporeal approach to the UD (12), despite this strategy does not improve complications rate (13).

The most important aspect in cancer surgery is oncological outcomes durability and, up to today, there has been paucity of studies comparing robot-assisted radical cystectomy (RARC) to open radical cystectomy (ORC).

Although Wei et al. recently suggested that recurrence patterns may be due to pre-existing micrometastases rather than pneumoperitoneum and tumor spillage (14), in the past years there have been concerns regarding higher rates of local recurrence in the minimally-invasive setting. Both Nguyen et al. (15) and Bochner et al. (6), in fact, observed an increased incidence of peritoneal carcinomatosis and extrapelvic lymph nodes involvement (though the difference was not statistically significant).

We recently proved that RARC does not increase the rate of positive surgical margins (4) and the lymph node yield [which significantly affects survival $(16,17)$ ] is not jeopardized by the minimally-invasive approach $(33.4 \mathrm{vs}$. 30.7; $\mathrm{P}=0.41$ ) (4). At 2 years follow-up, robotic and open cohorts displayed comparable recurrence-free survival (RFS) (87.8 vs. 84.4; $\log$ rank $\mathrm{P}=0.746$ ), cancer-specific survival (CSS) (89.6 vs. 88.3; $\log$ rank $\mathrm{P}=0.753)$ and overall survival (OS) rates (85.2 vs. 86; log rank $\mathrm{P}=0.909)$ (4). Also 5 years after surgery, oncologic outcomes of RARC appeared in line with historical open experiences (RFS, CSS and OS probabilities were $58 \% \pm 5 \%, 61 \% \pm 5 \%$ and $54 \% \pm 5 \%$, respectively) (18). Also RCTs recently confirmed that cancer control is not affected by the surgical approach, in the midterm (5-9). Still, there is a dearth of evidences supporting this equivalence in the long period. In 2014, Snow-Lisy et al. reported 10-year outcomes after minimally-invasive (laparoscopic and robotic) RC: RFS, CSS and OS were $54 \%, 63 \%$ and $35 \%$, respectively (19). Just recently, Hussein et al. provided a relevant contribution to the 
current literature publishing long-term results from the International Robotic Cystectomy Consortium: ten years after surgery, RFS was $59 \%$ while CSS and OS were $65 \%$ and $35 \%(20)$. Although the very first cases were also included in the analysis, such outcomes are consistent with those from large open series (21).

Then, most of the available studies support the equivalence of RARC and ORC in terms of morbidity and oncologic outcomes; the minimally invasive approach seems to overcome the conventional one only in terms of a reduced risk of transfusions and this might not be enough to justify its substantially increased costs (22).

Few authors, however, are investigating the role of nearinfrared fluorescence technology in enhancing anatomical structures identification thus potentially improving both safety and efficacy of RARC. In 2014 Manny and Hemal first reported on the feasibility of identification of sentinel node drainage (23). In the same year, Chopra et al. suggested to use the Firefly ${ }^{\circledR}$ module in assessing bowel vascularity to avoid mesenteric arcades injuries when isolating a segment of intestine for intracorporeal ileal neobladder construction (23). More recently, Ahmadi et al. resorted to intraoperative indocyanine green to assess the vascular integrity of the distal ureter before the ureteroenteric anastomosis was performed and observed that the use of this technology significantly reduced stricture rate (0\% vs. $10.6 \% ; \mathrm{P}=0.020)$ (23).

Concluding, based on the available evidences we can only state that ORC and RARC are broadly equivalent concerning safety and mid-term efficacy; future long term RCTs are needed to compare oncologic outcomes durability.

We are deeply persuaded that, to unveil the advantages of the robotic surgical system in the field of cystectomy, the concept of "replicating the open principles" should be abandoned and a comprehensive resort to its technology is mandatory (24).

\section{Acknowledgments}

None.

\section{Footnote}

Conflicts of Interest: The authors have no conflicts of interest to declare.

Ethical Statement: The authors are accountable for all aspects of the work in ensuring that questions related to the accuracy or integrity of any part of the work are appropriately investigated and resolved.

\section{References}

1. Alfred Witjes J, Lebret T, Compérat EM, et al. Updated 2016 EAU guidelines on muscle-invasive and metastatic bladder cancer. Eur Urol 2017;71:462-75.

2. Zamboni S, Soria F, Mathieu R, et al. Differences in trends in the use of robot-assisted and open radical cystectomy and changes over time in peri-operative outcomes among selected centres in North America and Europe: an international multicentre collaboration. BJU Int 2019. [Epub ahead of print].

3. Brassetti A, Möller A, Laurin O, et al. Evolution of cystectomy care over an 11-year period in a high-volume tertiary referral centre. BJU Int 2018;121:752-7.

4. Simone G, Tuderti G, Misuraca L, et al. Perioperative and mid-term oncologic outcomes of robotic assisted radical cystectomy with totally intracorporeal neobladder: results of a propensity score matched comparison with open cohort from a single-centre series. Eur J Surg Oncol 2018;44:1432-8.

5. Khan MS, Gan C, Ahmed K, et al. A single-centre early phase randomised controlled three-arm trial of open, robotic, and laparoscopic radical cystectomy (CORAL). Eur Urol 2016;69:613-21.

6. Bochner BH, Dalbagni G, Marzouk KH, et al. Randomized trial comparing open radical cystectomy and robot-assisted laparoscopic radical cystectomy: oncologic outcomes. Eur Urol 2018;74:465-71.

7. Messer JC, Punnen S, Fitzgerald J, et al. Health-related quality of life from a prospective randomised clinical trial of robot-assisted laparoscopic vs open radical cystectomy. BJU Int 2014;114:896-902.

8. Nix J, Smith A, Kurpad R, et al. Prospective randomized controlled trial of robotic versus open radical cystectomy for bladder cancer: perioperative and pathologic results. Eur Urol 2010;57:196-201.

9. Parekh DJ, Reis IM, Castle EP, et al. Robot-assisted radical cystectomy versus open radical cystectomy in patients with bladder cancer (RAZOR): an open-label, randomised, phase 3, non-inferiority trial. Lancet 2018;391:2525-36.

10. Novara G, De Marco V, Aragona M, et al. Complications and mortality after radical cystectomy for bladder transitional cell cancer. J Urol 2009;182:914-21.

11. Wilson TG, Guru K, Rosen RC, et al. Best practices 
in robot-assisted radical cystectomy and urinary reconstruction: recommendations of the Pasadena Consensus Panel. Eur Urol 2015;67:363-75.

12. Kimura S, Iwata T, Foerster B, et al. Comparison of perioperative complications and health-related quality of life between robot-assisted and open radical cystectomy: A systematic review and meta-analysis. Int J Urol 2019;26:760-74.

13. Lenfant L, Verhoest G, Campi R, et al. Perioperative outcomes and complications of intracorporeal vs extracorporeal urinary diversion after robot-assisted radical cystectomy for bladder cancer: a real-life, multiinstitutional French study. World J Urol 2018;36:1711-8.

14. Wei L, Hussein AA, Ma Y, et al. Accurate quantification of residual cancer cells in pelvic washing reveals association with cancer recurrence following robot-assisted radical cystectomy. J Urol 2019;201:1105-14.

15. Nguyen DP, Al Hussein Al Awamlh B, Wu X, et al. Recurrence patterns after open and robot-assisted radical cystectomy for bladder cancer. Eur Urol 2015;68:399-405.

16. Simone G, Papalia R, Ferriero M, et al. Stage-specific impact of extended versus standard pelvic lymph node dissection in radical cystectomy. Int J Urol 2013;20:390-7.

17. Simone G, Papalia R, Ferriero M, et al. Development and external validation of lymph node density cut-off points in prospective series of radical cystectomy and pelvic lymph node dissection. Int J Urol 2012;19:1068-74.

18. Brassetti A, Cacciamani G, Anceschi U, et al. Long-term oncologic outcomes of robot-assisted radical cystectomy

Cite this article as: Brassetti A, Mastroianni R, Simone G. Are we really seeking for equivalence?-The virtue of the robot is in technology. Transl Androl Urol 2019;8(Suppl 5):S502-S504. doi: 10.21037/tau.2019.08.19
(RARC) with totally intracorporeal urinary diversion (ICUD): a multi-center study. World J Urol 2019. [Epub ahead of print].

19. Snow-Lisy DC, Campbell SC, Gill IS, et al. Robotic and laparoscopic radical cystectomy for bladder cancer: longterm oncologic outcomes. Eur Urol 2014;65:193-200.

20. Hussein AA, Elsayed AS, Aldhaam NA, et al. Comparison of long-term oncologic outcomes among historical open and minimally invasive retrospective studies. J Urol 2019. [Epub ahead of print].

21. Hautmann RE, de Petriconi RC, Pfeiffer C, et al. Radical cystectomy for urothelial carcinoma of the bladder without neoadjuvant or adjuvant therapy: long-term results in 1100 patients. Eur Urol 2012;61:1039-47.

22. Leow JJ, Reese SW, Jiang W, et al. Propensitymatched comparison of morbidity and costs of open and robot-assisted radical cystectomies: a contemporary population-based analysis in the United States. Eur Urol 2014;66:569-76.

23. Cacciamani GE, Shakir A, Tafuri A, et al. Best practices in near-infrared fluorescence imaging with indocyanine green (NIRF/ICG)-guided robotic urologic surgery: a systematic review-based expert consensus. World J Urol 2019. [Epub ahead of print].

24. Simone G, Papalia R, Misuraca L, et al. Robotic intracorporeal padua ileal bladder: surgical technique, perioperative, oncologic and functional outcomes. Eur Urol 2018;73:934-40. 